June 15, 2018

FTUV/96-07

IFIC/96-08

\title{
An improved cosmological bound on the tau-neutrino mass
}

\author{
A.D. Dolgov 円, S. Pastor, and J. W. F. Valle ? \\ Instituto de Física Corpuscular - C.S.I.C. \\ Departament de Física Teòrica, Universitat de València \\ 46100 Burjassot, València, SPAIN
}

\begin{abstract}
We consider the influence of non-equilibrium electronic neutrinos (and anti-neutrinos) on the neutron-to-proton ratio. These neutrinos would come from massive $\nu_{\tau}$ annihilations $\bar{\nu}_{\tau} \nu_{\tau} \rightarrow \bar{\nu}_{e} \nu_{e}$. For sufficiently large $\nu_{\tau}$ masses this new effect would strongly enhance the (n/p)-ratio, leading to a very stringent bound on the $\nu_{\tau}$ mass, even adopting a rather weak upper bound on the effective number on neutrino species during nucleosynthesis.
\end{abstract}

\footnotetext{
${ }^{1}$ Permanent address: ITEP, 113259, Moscow, Russia.
}

${ }^{2}$ E-mail valle@flamenco.ific.uv.es 
The tau-neutrino remains the only one which still can have mass in the $\mathrm{MeV}$ range. This possibility is theoretically viable, since it can be unstable on cosmological time scales, thus avoiding the limits set by the relic density [1]. It is also quite interesting experimentally, due to the good prospects for improving the $\nu_{\tau}$ mass limits at a $\mathrm{B}$ meson or tau-charm factory [2]. In addition, an $\mathrm{MeV}$ tau neutrino can be quite interesting cosmologically [3].

The present-day experimental limit on its mass is [4]]:

$$
m_{\nu_{\tau}}<23 \mathrm{MeV}
$$

Primordial nucleosynthesis considerations [5] rule out $\nu_{\tau}$ masses in the range [6, 7]:

$$
0.5 \mathrm{MeV}<m_{\nu_{\tau}}<35 \mathrm{MeV}
$$

if $\nu_{\tau}$ is stable on the nucleosynthesis time scale and does not possess new interactions capable of depleting its density in the cosmic plasma [ Calculations of spin-flip processes for the case of a Dirac-type $\nu_{\tau}$ shift the lower bound in this interval down to $0.2 \mathrm{MeV}$ [8]. Altogether these bounds can be summarised as $m_{\nu_{\tau}}^{D}<0.2 \mathrm{MeV}$ and $m_{\nu_{\tau}}^{M}<0.5 \mathrm{MeV}$, where "M" and "D" stands for the Dirac and Majorana mass respectively.

All these bounds are obtained under assumption that the effective number of extra neutrino species $\Delta k_{\nu}$ during nucleosynthesis cannot be larger than 0.4 or 0.6. Recent contradictory data on the primordial deuterium abundance [9, 10] may cast some doubts on the validity of this limit (for recent analysis see refs. 11]). In particular, if $\Delta k_{\nu}=1$ is allowed, there may be an open window for neutrino mass somewhere near $20 \mathrm{MeV}$.

In this letter we will show however that this is not the case, at least for a Diractype neutrino, and that high $\nu_{\tau}$ mass values are excluded even when we adopt a much weaker upper bound on the extra number of neutrino species. Our result follows from the consideration of non-equilibrium $\nu_{e}$ 's and $\bar{\nu}_{e}$ 's originating from the annihilation of heavy $\nu_{\tau}$ 's. The effect of non-equilibrium electronic neutrinos on the kinetics of (n-p)-reactions was considered in refs. [12], for the case where the $\nu_{e}$ 's and $\bar{\nu}_{e}$ 's arise from heavy particle decays during nucleosynthesis. Nonequilibrium $\nu_{e}$ 's and $\bar{\nu}_{e}$ 's produced by $e^{+} e^{-}$-annihilation in the standard model also change the (n/p)-ratio but the effect is small [13]. In contrast, the effect we

\footnotetext{
${ }^{3}$ Of course such interactions are needed, at some level, in order to comply with the limit on the relic neutrino density, but the time scale involved is much larger.
} 
consider here is much more significant. For example, for the case of a very heavy $m \sim 20 \mathrm{MeV} \nu_{\tau}$ we show that it is equivalent to 0.8 extra massless neutrinos species for the case of a Dirac $\nu_{\tau}$, and to 0.1 extra neutrinos for the Majorana case. The importance of this effect was mentioned in ref.[0] but the calculations were not performed there because with the bound $\Delta k_{\nu}<0.6$ the complete range of high $\nu_{\tau}$ mass values was already excluded even without this effect.

One can understand the essential features of the relevant phenomena in the following way. At some stage $\nu_{\tau}$ 's, being massive, went out of thermal equilibrium. Their number density tends to a "frozen" value, but their residual annihilation produced energetic non-equilibrium $\nu_{e}$ 's and $\bar{\nu}_{e}$ 's. If this take place sufficiently late, roughly speaking, when $T \lesssim 2 \mathrm{MeV}$, the produced non-equilibrium $\nu_{e}$ and $\bar{\nu}_{e}$ are not thermalized. The resulting distortion of the $\nu_{e}$ spectrum would shift the frozen (n/p)-ratio and, correspondingly, the primordial ${ }^{4} \mathrm{He}$ abundance. The effect has different signs for high and low energy $\nu_{e}$ 's. If there is an excess of $\nu_{e}$ 's ( or $\bar{\nu}_{e}$ 's) at the high energy tail of the spectrum, the (n/p)-ratio increases. Indeed, this ratio is determined by the competition between the universe expansion rate and the rates of the reactions:

$$
\begin{aligned}
& p+e^{-} \leftrightarrow n+\nu_{e} \\
& n+e^{+} \leftrightarrow p+\bar{\nu}_{e}
\end{aligned}
$$

An excess of energetic $\nu_{e}$ 's and $\bar{\nu}_{e}$ 's destroys neutrons in the first reaction and produces them in the second one, with the same rate. However, since the number density of protons at the moment of freezing of these reactions is about 6 times larger than that of neutrons, the second process is more efficient and we get more neutrons than in the standard case. On the other hand if there is an excess of neutrinos at low energy, its effect on the process (目) is small because of the threshold effect. However, it enhances the destruction of neutrons in the reaction (3) and, correspondingly, the (n/p)-ratio goes down. There is also another effect related to the increased overall number density of electronic neutrinos coming from the $\bar{\nu}_{\tau} \nu_{\tau} \rightarrow \bar{\nu}_{e} \nu_{e}$ annihilation. This effect decreases the (n/p)-freezing temperature as well as the (n/p)-ratio independently of the energies of the electronic neutrinos. However, as we will see in what follows, the spectrum distortion at high energies is more important and the net result of all these effects is to enhance the $(\mathrm{n} / \mathrm{p})$-ratio leading to more stringent $\nu_{\tau}$ mass limits.

Though we obtained our results numerically, an accurate analytical treatment 
is possible for large values of the $\nu_{\tau}$ mass $m_{\nu \tau}>10 \mathrm{MeV}$. The kinetic equation which governs the spectrum of electronic neutrinos has the form:

$$
\begin{array}{r}
\frac{\partial f_{e 1}}{\partial t}-H \omega_{1} \frac{\partial f_{e 1}}{\partial \omega_{1}}=\frac{1}{2 \omega_{1}} \int \frac{d^{3} p_{1}}{(2 \pi)^{3} 2 E_{1}} \frac{d^{3} p_{2}}{(2 \pi)^{3} 2 E_{2}} \frac{d^{3} k_{2}}{(2 \pi)^{3} 2 \omega_{2}} \\
(2 \pi)^{4} \delta^{4}\left(p_{1}+p_{2}-k_{1}-k_{2}\right) \sum\left|A^{2}\right|\left(f_{\tau 1} f_{\tau 2}-f_{e 1} f_{e 2}\right)
\end{array}
$$

where $f_{\tau}$ and $f_{e}$ are the $\nu_{\tau}$ and $\nu_{e}$ spectral functions (or occupation numbers), respectively, and $H$ is the Hubble parameter. If the expansion is dominated by photons, $e^{-}, e^{+}$, and two massless neutrino species, the effective number of degrees of freedom is $g_{*}=9$ so that $H \approx 5 T^{2} / m_{P l}$. As a first approximation we take into account only the reaction $\nu_{\tau 1}+\bar{\nu}_{\tau 2} \leftrightarrow \nu_{e 1}+\bar{\nu}_{e 2}$ in the collision integral. Here $A$ is the amplitude of this reaction and the summation is made over the spins of all particles except for $\nu_{e 1}$. For the case of a Dirac $\nu_{\tau}$ one has

$$
\sum\left|A_{D}^{2}\right|=32 G_{F}^{2}\left(p_{1} k_{1}\right)^{2}
$$

while for the Majorana case:

$$
\sum\left|A_{M}^{2}\right|=16 G_{F}^{2}\left[\left(p_{1} k_{1}\right)^{2}+\left(p_{2} k_{1}\right)^{2}-m^{2}\left(k_{1} k_{2}\right)\right]
$$

where the identical particle factor $1 / 2$ ! has already been included in (7).

The other relevant processes temporarily omitted in the right hand side (r.h.s.) of eq.(5), namely elastic scattering of $\nu_{e}\left(\bar{\nu}_{e}\right)$ on themselves and other light fermions are essential at higher temperatures. They smooth down spectral distortions and force the distribution back into equilibrium, somewhat diminishing the effect. We will take them into account in what follows (see eqs.(9, 101)).

We will solve equation (5) (or equivalently eqs.(9, 10)) perturbatively assuming that $f_{e}$ has the form $f_{e}=f_{e}^{(e q)}+\delta f$, where for the equilibrium part we take the Boltzmann expression $f_{e}^{(e q)}=\exp (-\omega / T)$. Quantum statistics corrections may diminish the effect by (5-10)\% [14]. We neglect terms of the second order in $\delta f$. The distortion of the electronic neutrino spectrum is induced by the deviation of the massive $\nu_{\tau}$ from thermal equilibrium which arises when the temperature of the cosmic plasma drops below the $\nu_{\tau}$ mass. It is usually assumed that kinetic equilibrium is maintained, while an effective chemical potential of the same magnitude for particles and antiparticles is generated [15]. In other words $f_{\tau}$ takes the form $f_{\tau}=\exp (\xi(t)-E / T)$. The $\nu_{\tau}$ number density is calculated from the well known equation:

$$
\dot{n}_{\tau}+3 H n_{\tau}=\langle\sigma v\rangle\left(n_{\tau}^{(e q) 2}-n_{\tau}^{2}\right)
$$


where $\langle\sigma v\rangle$ is the thermally averaged cross-section of $\nu_{\tau}$-annihilation (multiplied by velocity) and $n_{\tau}^{(e q)}$ is their equilibrium number density. We write $n_{\tau}$ as usual, in the form $n_{\tau}=r n_{0}$ where $n_{0} \approx 0.09 g_{s} T^{3}$ is the equilibrium number density of massless neutrinos with the same temperature and $g_{s}$ is the spin counting factor. We have calculated $r$ numerically solving eq.(8) and parametrised the solution in the form $r=r_{0}+r_{1} m_{\nu_{\tau}} / T$. The results we obtain agree with those of ref. [7].

We assume that the temperature drops in the usual way, $\dot{T}=-H T$, possible corrections to this law are not essential here. In this case the equilibrium part of $f_{e}$ annihilates the r.h.s. of eq.(5) and it would contain only $\delta f$. Introducing new variables $x \equiv m / T$ and $y \equiv \omega / T$ we can rewrite equation (5) in the form:

$H x \frac{\partial \delta f_{D}}{\partial x}=\frac{2 G_{F}^{2} m^{5}}{3 \pi^{3}}\left(\frac{n_{\tau}^{2}}{n_{\tau}^{(e q) 2}}-1\right) \frac{\exp (-y-\alpha)}{x^{2} y^{1 / 2}} \int_{0}^{\infty} d u e^{-u}\left(\frac{3}{4}+\frac{u}{\alpha}\right) \sqrt{u\left(1+\frac{u}{\alpha}\right)}+\ldots$

for the Dirac case while, for the Majorana case we obtain

$$
H x \frac{\partial \delta f_{M}}{\partial x}=\frac{2 G_{F}^{2} m^{5}}{3 \pi^{3}}\left(\frac{n_{\tau}^{2}}{n_{\tau}^{(e q) 2}}-1\right) \frac{\exp (-y-\alpha)}{x^{4} y^{-1 / 2}} \int_{0}^{\infty} d u e^{-u} u^{3 / 2} \sqrt{1+\frac{u}{\alpha}}+\ldots
$$

Here $\alpha \equiv x^{2} / y$ and the dots denote contributions from elastic scattering which we have so far omitted.

In the limit of high $\nu_{\tau}$ mass, i.e. for $x \gg 1$ and $\alpha \gg 1$, the integrals can be performed approximately so that the r.h.s. of these equations can be written as:

$$
\frac{\partial \delta f}{\partial x} \approx 0.96 \times 10^{-2} m^{3} \frac{r^{2}-r^{(e q) 2}}{x^{4} y^{1 / 2}} \exp \left[-\frac{(x-y)^{2}}{y}\right] F_{D}(x, y)+\ldots
$$

(Dirac case),

$$
\frac{\partial \delta f}{\partial x} \approx 1.92 \times 10^{-2} m^{3} \frac{r^{2}-r^{(e q) 2}}{x^{6} y^{-1 / 2}} \exp \left[-\frac{(x-y)^{2}}{y}\right] F_{M}(x, y)+\ldots
$$

(Majorana case).

Here and in what follows $m$ is measured in $\mathrm{MeV}, F_{D}=\left(1+11 y / 4 x^{2}+\right.$ $\left.65 y^{2} / 32 x^{4}\right) /\left(1+15 / 4 x+165 / 32 x^{2}\right)$, and $F_{M}=\left(1+5 y / 4 x^{2}-35 y^{2} / 32 x^{4}\right) /(1+$ $\left.15 / 4 x+165 / 32 x^{2}\right)$. The numerators come from the expansion of the integrals in the r.h.s. of equations $(9,10)$ and the denominators come from the non-relativistic expansion of the number density.

It is straightforward to take into account the other processes where $\nu_{e}$ and $\bar{\nu}_{e}$ participate, denoted by "..." in the above equations. They are either elastic scattering off light leptons, $\nu_{e}+l \leftrightarrow \nu_{e}+l$, or $\nu_{e}+\bar{\nu}_{e} \leftrightarrow l+\bar{l}$ annihilations. These processes tend to restore equilibrium, thus diminishing the distortion $\delta f$. 
The collision integral in the r.h.s. of eq. (5) contains $\delta f$ in terms of two types: first, when $\delta f=\delta f\left(k_{1}\right)$ refers to the particle under consideration and, second, when $\delta f=\delta f\left(k_{2}\right)$ refers to one over whose momentum we integrate in $d^{3} k_{2}$. The first ones come with a negative sign and force the system into equilibrium, while the terms of the second type typically appear with positive sign and induce a deviation from equilibrium f In order to account for the terms of the second kind one has to solve a complicated integro-differential kinetic equation. In contrast it is very easy to treat the terms of the first kind. They result in the addition of an extra term of the form $(-\delta f) F(x, y)$, with a known function $F(x, y)$, to the r.h.s. of eqs.(9, 10). In what follows we take into account only the contribution of the terms of the first kind, thus somewhat overestimating the suppression factor. The real effect should be somewhat larger. However, even in this approximation our effect results in a considerable improvement of the strength of the bound on $m_{\nu_{\tau}}$.

Taking into account all possible reactions of $\nu_{e}$ with light leptons we get $F \approx m^{3} y / 3 x^{4}$. The result is valid for arbitrary $x$ and $y$ values (the restriction $x \gg 1$ is not assumed here). After a simple algebra we get the following equations for the spectrum distortion:

$$
\begin{aligned}
\frac{\partial \delta f_{D}}{\partial x} & =0.0096 m^{3} \frac{r^{2}-r^{(e q) 2}}{x^{4} y^{1 / 2}} \exp \left(-\frac{(x-y)^{2}}{y}\right) F_{D}(x, y)-\frac{m^{3} y}{3 x^{4}} \delta f_{D} \\
\frac{\partial \delta f_{M}}{\partial x} & =0.0192 m^{3} \frac{r^{2}-r^{(e q) 2}}{x^{6} y^{-1 / 2}} \exp \left(-\frac{(x-y)^{2}}{y}\right) F_{M}(x, y)-\frac{m^{3} y}{3 x^{4}} \delta f_{M}
\end{aligned}
$$

where "M" and "D" stand for the Dirac and Majorana cases, respectively.

The solutions of these equations can be easily written. These expressions should be substituted into the equation which governs the neutron-to-proton ratio. Since the spectrum is distorted at high neutrino energies we may neglect the electron mass, leading to the approximate form (see e.g. ref.[13]):

$$
\begin{gathered}
\frac{d r_{n}}{d T}=-0.05 T^{2}\left[\left(24+12 \beta+2 \beta^{2}\right)\left(e^{-\beta}-r_{n}\left(1+e^{-\beta}\right)\right)+\right. \\
\left.(1 / 2) \int_{0}^{\infty} d y y^{2}(y+\beta)^{2}\left(\delta f(y+\beta)-r_{n}(\delta f(y)+\delta f(y+\beta))\right)\right]
\end{gathered}
$$

Here $r_{n}$ is the ratio of the neutron number density to the total baryonic number density. The (n/p)-ratio is expressed through $(n / p)=r_{n} /\left(1-r_{n}\right)$, and $\beta \equiv \Delta m / T$

\footnotetext{
${ }^{4}$ The terms of the second kind may also appear with a negative sign, like e.g. in the process $\nu_{e}+\nu_{e} \leftrightarrow \nu_{e}+\nu_{e}$ from neutrinos in the initial state, but the contribution from similar terms coming from neutrinos in the final state over-weights the negative one.
} 
where $\Delta m=1.3 \mathrm{MeV}$ is the neutron-proton mass difference. Of course, in our numerical integration we have used the exact equation for the (n/p)-ratio as given e.g. in ref. [13].

We can now roughly estimate the variation of (n/p)-ratio using eq.(15) in the following way. First, we integrate eqs. (13,14) neglecting the last term in the r.h.s. In this approximation the $\nu_{e}$ 's and $\bar{\nu}_{e}$ 's are in thermal equilibrium above $T \sim 2 \mathrm{MeV}$, while below this temperature the elastic scattering processes are not important. We estimate the relevant integral over $x$ in the Gaussian approximation near $x=y$ and integrate the obtained result over $y$ as written in eq.(15). So this equation takes the form:

$$
\begin{aligned}
& \frac{d r_{n}}{d T}=-0.05 T^{2}\left[\left(24+12 \beta+2 \beta^{2}\right) e^{-\beta}+\Delta^{-} / 2-\right. \\
& \left.r_{n}\left(\left(24+12 \beta+2 \beta^{2}\right)\left(1+e^{-\beta}\right)+\Delta^{+} / 2+\Delta^{-} / 2\right)\right]
\end{aligned}
$$

where

$$
\begin{aligned}
& \Delta_{D}^{ \pm} \approx 0.017 r_{D}^{2} m^{3} x\left(1-\frac{x_{i}}{x} \pm \frac{2 \Delta m}{m} \ln \frac{x}{x_{i}}\right) \\
& \Delta_{M}^{ \pm} \approx 0.034 r_{M}^{2} m^{3}\left(\ln \frac{x}{x_{i}} \pm 2 \beta\left(\frac{1}{x_{i}}-\frac{1}{x}\right)\right)
\end{aligned}
$$

with $x_{i}=m / T_{i}=m / 2$. The signs "+" and "-" in the above expressions correspond respectively to the integrals $\int d y y^{2}(y+\beta)^{2} \delta f(y)$ and to $\int d y y^{2}(y+$ $\beta)^{2} \delta f(y+\beta)$.

As we have already noted, the modification of the neutrino spectrum results in two opposite effects. First, the "equilibrium" value of $r_{n}$ (obtained by equating the r.h.s. to zero) increases. Second, the (n-p)-reactions get frozen later, as the excess of energetic $\nu_{e}$ 's and $\bar{\nu}_{e}$ 's increases their rates. Taking into account both effects we can write the correction to $r_{n}$ as:

$$
\frac{\delta r_{n}}{r_{n}}=\left[1+\frac{\Delta^{-} e^{\beta_{1}}-\left(\Delta^{+}+\Delta^{-}\right) /\left(1+e^{-\beta_{1}}\right)}{48+24 \beta_{1}+4 \beta_{1}^{2}}\right] e^{\beta_{0}-\beta_{1}} \frac{1+e^{-\beta_{0}}}{1+e^{-\beta_{1}}}-1
$$

where $\beta_{0} \equiv \Delta m / T_{f 0}, T_{f 0}=0.65 \mathrm{MeV}$ is the standard value of (n-p)-freezing temperature and $\Delta^{ \pm}$are taken at $x=m / T_{f 0}$. The new freezing temperature $T_{f 1}$ can be found from

$$
\beta_{1}=\beta_{0}+\frac{\beta_{0}^{4}}{\left(48+24 \beta_{0}+4 \beta_{0}^{4}\right)\left(1+e^{-\beta_{0}}\right)} \int_{\beta_{0}}^{\infty} d w \frac{\Delta^{+}+\Delta^{-}}{w^{4}}
$$

Using these expressions we obtain $\delta r_{n} / r_{n}=(5-10) \%$ for a Dirac $\nu_{\tau}$ with mass in the range $m=10-20 \mathrm{MeV}$, while for the Majorana case we find $\delta r_{n} / r_{n}=(1-$ 
2)\%. These results are in reasonable agreement with our numerical calculations described below.

Now let us briefly describe the calculations. The solution of eqs.(13, 14) was taken analytically in terms of one-dimensional integral $\int_{0}^{x} d x^{\prime}$. Then we substituted this expression into the exact equation governing the $(\mathrm{n} / \mathrm{p})$-ratio. Integration over $y$ in this equation can be approximately done analytically with a very good precision. The last integral over $x^{\prime}$ was performed numerically for different values of $x$ and accurate interpolating functions were substituted into the equation for the $(\mathrm{n} / \mathrm{p})$-ratio. This first order differential equation was solved numerically.

In the case of Dirac $\nu_{\tau}$ we obtained $\delta r_{n} / r_{n}=4.2 \%$ for $m=10 \mathrm{MeV}$ and $3.6 \%$ for $m=20 \mathrm{MeV}$. In the Majorana case we got $1.2 \%$ for $10 \mathrm{MeV}$ and $0.5 \%$ for $20 \mathrm{MeV}$. These results can be rewritten as corrections to the effective neutrino number $N_{e q}$ as a function of the $\nu_{\tau}$ mass as shown in the figure. The solid curves denote the results we obtain including the effect of non-equilibrium electronic neutrinos and antineutrinos from massive $\nu_{\tau}$ annihilations on the neutron-toproton ratio. The dashed curves represent the corresponding results neglecting these corrections [6, 7]. As can be seen from the figure the increase of $N_{e q}$ in the mass range of interest is larger for the case of Dirac than it is for the Majorana neutrino case. From our results one concludes that relatively long-lived massive tau neutrinos above the few $\mathrm{MeV}$ range are ruled out by the requirement $N_{e q} \leq 4$. Thus it would seem from our results that the only way to accommodate a massive tau neutrino above the few $\mathrm{MeV}$ range is if there are new interactions beyond those of the standard model that can make it decay and/or annihilate efficiently on the nucleosynthesis time scale. The case of unstable $\nu_{\tau}$ 's has been considered in refs. [16]. The effect we have discussed would also improve the bounds obtained in these papers. The alternative case of neutrinos with large majoron annihilation cross sections is under consideration [17].

As this work was completed we became aware of the paper [18] where the nonequilibrium heating of electronic neutrinos by $\nu_{\tau} \bar{\nu}_{\tau}$-annihilation was considered. The conclusion of this paper about the influence of non-equilibrium $\nu_{e}$ 's and $\bar{\nu}_{e}$ 's on the $(\mathrm{n} / \mathrm{p})$-ratio is opposite to ours. We think that the results differ because these authors took into account only a decrease of the (n-p)-freezing temperature due to the excess of electronic neutrinos, but neglected the spectrum distortion which we have discussed here and which turns out to have a stronger impact on 
the $(\mathrm{n} / \mathrm{p})$-ratio.

This work was supported by DGICYT under grants PB92-0084 and SAB940089 (A. D.). S.P. was supported by Conselleria d'Educació i Ciència of Generalitat Valenciana.

\section{References}

[1] For recent reviews see J. W. F. Valle, Gauge Theories and the Physics of Neutrino Mass, Prog. Part. Nucl. Phys. 26 (1991) 91-171 (ed. A. Faessler); and G. Gelmini and S. Roulet, UCLA/94/TEP/36 and references therein.

[2] J. Gómez-Cadenas, M. C. González-García, Phys. Rev. D39 (1989) 1370; J. Gómez-Cadenas et al., Phys. Rev. D41 (1990) 2179; R. Alemany et al in ECFA/93-151, ed. R. Aleksan, A. Ali, p. 191-211, hep-ph/9307252.

[3] J. Bardeen, J. Bond and G. Efstathiou, Astrophys. J. 321 (1987) 28; J. Bond and G. Efstathiou, Phys. Lett. B265 (1991) 245; M. Davis et al., Nature 356 (1992) 489; S. Dodelson, G. Gyuk and M. Turner, Phys. Rev. Lett. 72 (1994) 3754; H. Kikuchi and E. Ma, Phys. Rev. D51 (1995) 296; H. B. Kim and J. E. Kim, Nucl. Phys. B433 (1995) 421; M. White, G. Gelmini and J. Silk, Phys. Rev. D51 (1995) 2669; A. S. Joshipura and J. W. F. Valle, Nucl. Phys. B440 (1995) 647.

[4] D. Buskulic et al., Phys. Lett. B349 (1995) 585.

[5] For a recent review see S. Sarkar, Oxford preprint OUTP-95-16P

[6] E. Kolb, M.S. Turner, A. Chakravorty and D.N. Schramm, Phys. Rev. Lett. 67 (1991) 533.

[7] A.D. Dolgov and I.Z. Rothstein, Phys. Rev. Lett. 71 (1993) 476.

[8] A.D. Dolgov, K. Kainulainen and I.Z. Rothstein, Phys. Rev. D51 (1995) 4129; for earlier work see G.M. Fuller and R.A. Malaney, Phys. Rev. D43 (1991) 3136; W.P. Lam and K.-W. Ng, Phys. Rev. D44 (1991) 3345; K. Enqvist and H. Uibo, Phys. Lett. B301 (1993) 376.

[9] R.F. Carswell, M. Rauch R.J. Weynman et al., MNRAS 268 (1994) L1; A. Songalia, L.L. Cowie, C. Hogan and M. Rugers, Nature 368 (1994) 599.

[10] D. Tytler and X.M. Fan, Bull.Am.Astr.Soc. 26 (1994) 1424. 
[11] K.A. Olive and G. Steigman Astrophys. J. Suppl. 97 (1995) 49; K.A. Olive and S.T. Scully, UMN-TH-1341/95, to be published in Int. J. Mod. Phys. A; C.J. Copi, D.N. Schramm and M.S. Turner, Science 267 (1995) 192.

[12] R.J. Scherrer, MNRAS 210 (1984); N. Terasawa and K. Sato, Phys. Lett. B185 (1988) 412; A.D. Dolgov and D.K. Kirilova, Int. J. Mod. Phys. A3 (1988) 267; G. Gyuk and M.S. Turner, Phys. Rev. D50 (1994) 6130.

[13] A.D. Dolgov and M. Fukugita Pisma ZhETF 56 (1992) 129 (English translation: JETP Letters 56 (1992) 123); Phys. Rev. D46 (1992) 5378; S. Dodelson and M.S. Turner, Phys. Rev. D46 (1992) 3372; S. Hannestad and J. Madsen, Phys. Rev. D52 (1995) 1764.

[14] A.D. Dolgov and K. Kainulinen, Nucl. Phys. B402 (1993) 349.

[15] A.D. Dolgov, Sov. J. Nucl. Phys. 32 (1980) 1606.

[16] M. Kawasaki, P. Kernan, H.-S. Kang, R.J. Scherer, G. Steigman and T.P. Walker, Nucl. Phys. B419 (1994) 105; S. Dodelson, G. Gyuk and M.S. Turner, Phys. Rev. D49 (1994) 5068; for a review see G. Steigman; proceedings of the International School on Cosmological Dark Matter, (World Scientific, 1994), ed. J. W. F. Valle and A. Perez, p. 55

[17] A.D. Dolgov, S. Pastor, J. Romão, and J.W.F. Valle (work in preparation).

[18] B.D. Fields, K. Kainulainen and K.A. Olive, CERN-TH/95-335, UMN-TH1417/95, hep-ph/9512321. 


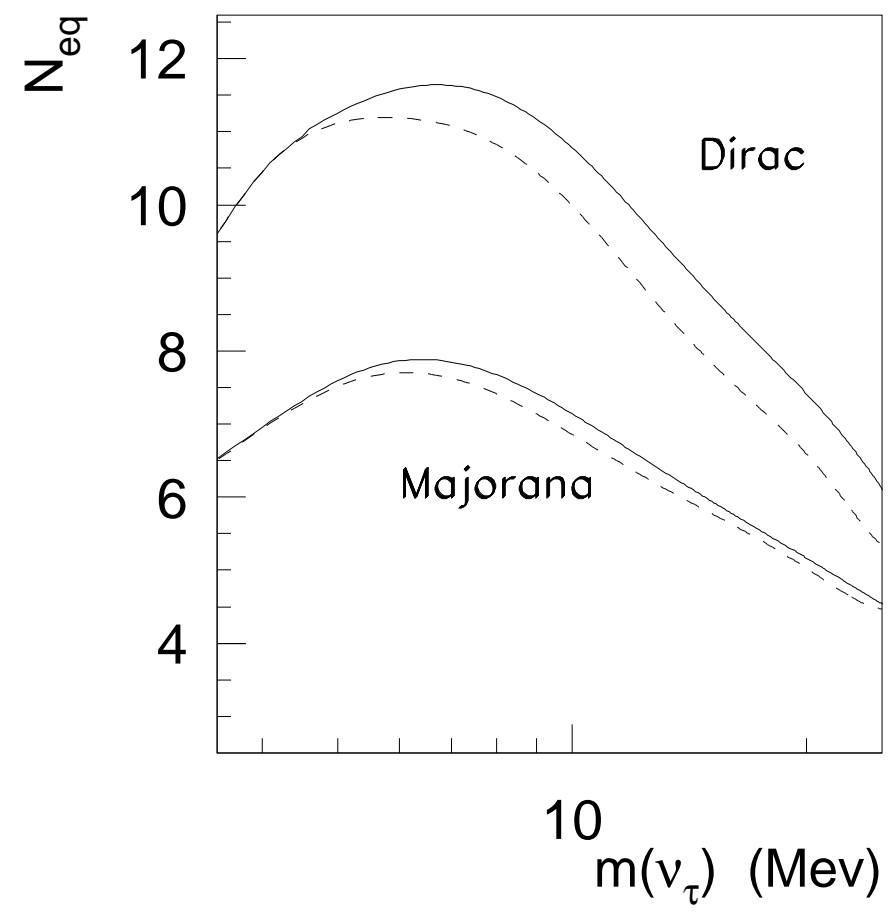

